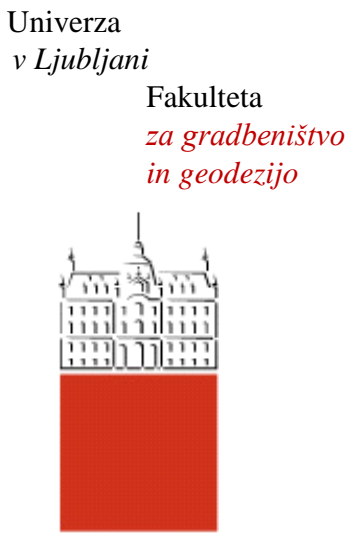

Jamova cesta 2

1000 Ljubljana, Slovenija

http://www3.fgg.uni-lj.si/

\section{DRUGG - Digitalni repozitorij UL FGG http://drugg.fgg.uni-lj.si/}

Ta članek je avtorjeva zadnja recenzirana različica, kot je bila sprejeta po opravljeni recenziji.

Prosimo, da se pri navajanju sklicujte na bibliografske podatke, kot je navedeno:
University
of Ljubljana

Faculty of Civil and Geodetic Engineering

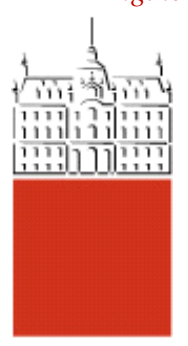

Jamova cesta 2 SI - 1000 Ljubljana, Slovenia http://www3.fgg.uni-lj.si/en/

DRUGG - The Digital Repository http://drugg.fgg.uni-lj.si/

This version of the article is author's manuscript as accepted for publishing after the review process.

When citing, please refer to the publisher's bibliographic information as follows:

Zupan, D., Saje, M. 2004. On "A proposed standard set of problems to test finite element accuracy": the twisted beam. Finite elements in analysis and design 40: 1445-1451. DOI: 10.1016/j.finel.2003.10.001. 


\title{
On "A proposed standard set of problems to test finite element accuracy": The twisted beam
}

\author{
D. Zupan, M. Saje* \\ University of Ljubljana, Faculty of Civil and Geodetic Engineering, Jamova 2, \\ SI-1115 Ljubljana, Slovenia
}

\begin{abstract}
The standard test problem of MacNeal and Harder [1] for the verification of spatial beam finite elements, i.e. the deflection of the initially twisted beam, is commented through the analysis of three variants of initially twisted beams: (i) a linearly twisted beam with a constant cross-section, (ii) a non-linearly twisted beam with a constant cross-section, and (iii) a non-linearly twisted beam with variable cross-sections. Our numerical results lead to the conclusion that the twisted beam problem [1] assumes the linearly twisted, curved-edge beam.
\end{abstract}

Key words: three-dimensional beam, finite element method, standard test problem.

\section{Introduction}

The initially twisted cantilever beam is one of the standard test problems for verifying the finite-element accuracy (MacNeal and Harder [1]). The beam is clamped at one end and loaded either with unit in-plane or unit out-of-plane force at the other. The centroidal axis of the beam is straight at the undeformed configuration, while its cross-sections are twisted about the centroidal axis from 0 at the clamped end to $\frac{1}{2} \pi$ at the free end. Other geometric and material characteristics of the beam are: beam length $L=12$, width of the cross-section $h=1.1$, its thickness $t=0.32$, Young's modulus $E=29 \cdot 10^{6}$, and Poisson's ratio $\nu=0.22$. (Since the plane of a twisted beam is not uniquely described, we consider the plane $x z$ as the plane of the beam (see Figure 1)).

* Corresponding author. Tel.: +38614768 613; FAX: +38614768629

Email address: msaje@fgg.uni-lj.si (M. Saje). 
The aim of the test is to find out if a finite element is capable of considering the initially non-planar configuration of the beam properly. Although the test is interesting for practical applications and in assessing the beam element accuracy, it has rarely been used by beam-element developers. A precise comparison of their numerical results based on the beam theory seems only to have been given by Dutta and White [2]. On the other hand, the test has been widely used by shell-element developers, see e.g. [3-9], among many others, and by 3D element developers, e.g. by Lo and Ling [10] who used the test to examine their 10-node tetrahedral element.

It appears, however, that the authors [2-10] used a pre-twisted beam with straight edges (as illustrated in Figure 1b) and the non-linear variation of the pre-twist along the axis, while we believe that MacNeal and Harder [1] considered a beam with curved edges - compare their Figure 6 with our analogous Figure 1a, and observe that, as cited on page 11 of [1], "the warp of each element is only $7.5^{\circ}$ ". Their finite-element mesh consists of 12 equal-length elements in axial direction. As $12 \cdot 7.5^{\circ}=90^{\circ}$, it can only be concluded that the pre-twist in [1] varies linearly along the axis. In the following we first show that the way in which the initial, pre-twisted geometry of the beam is modeled, considerably influences the numerical results.

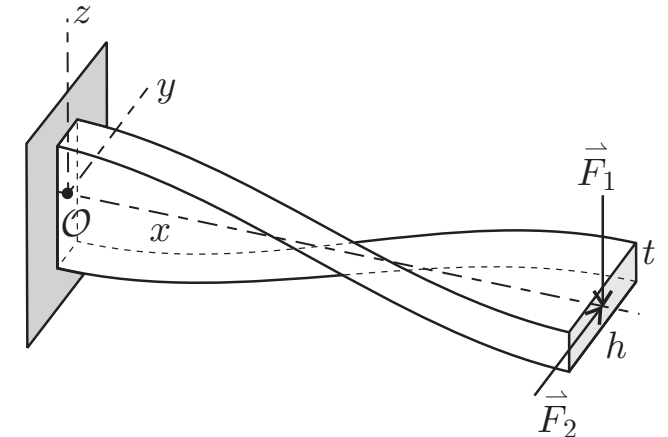

a)

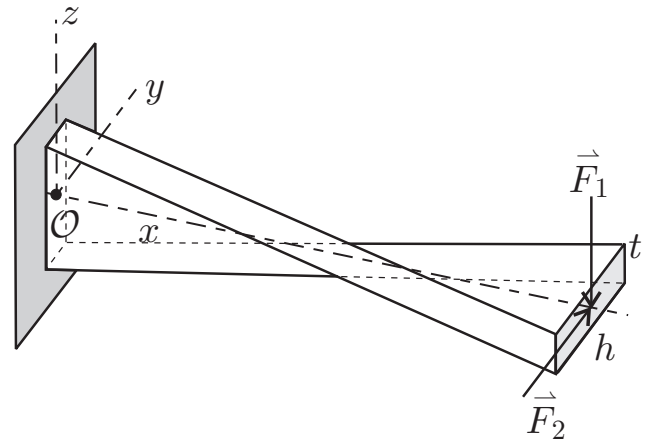

b)

Figure 1. Two natural options for a beam initially twisted for $\pi / 2$ : a) a linear pre-twist along the beam: curved edges, and b) non-linear pre-twist: straight edges.

\section{The variation of the pre-twist angle along the axis of the beam}

There are two natural kinds of pre-twists. In the first one, the initial twist angle, $\psi$, is taken to be the linear function of the arc-length, $x$, of the line of centroids:

$$
\psi(x)=\frac{\pi}{2 L} x
$$


the related twisted shape of the undeformed cantilever has curved edges and is illustrated in Figure 1a. Such a shape is typical for various turbine blades.

The second kind of a pre-twist assumes a non-linear dependence between the pre-twist angle and the arc-length parameter. The simplest example of a nonlinearly pre-twisted beam is a beam with straight edges (see Figure 1b). This kind of the pre-twist seems to be considered by the cited finite-element developers $([2-10])$. Please see their illustrations, but note that no explicit statement concerning the variation of initial twist is made in their texts. Although the straight-edge shape looks rather simple, the relation between $\psi$ and $x$ is neither simple nor self-evident. We will now derive this relation. We may assume that the thickness of the cross-section can be neglected: $t=0$. A 4 -node surface with straight edges is then obtained, as shown in Figure 2a.

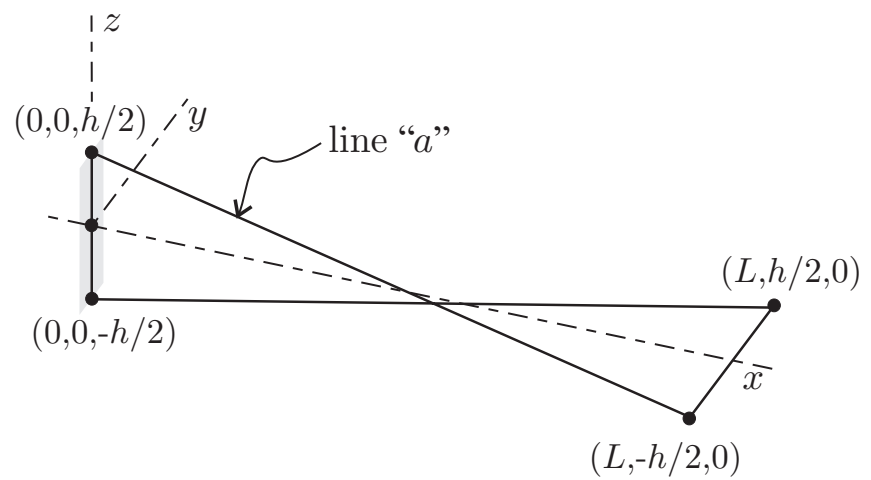

a)

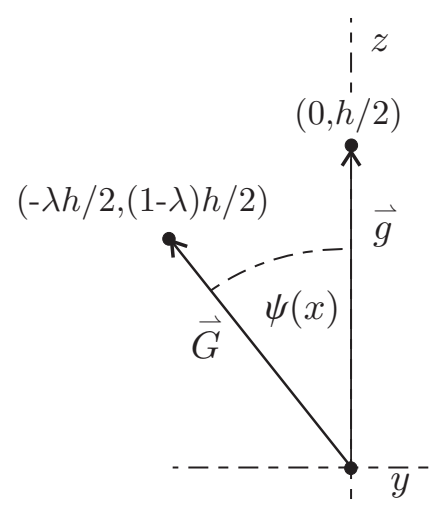

b)

Figure 2. Beam with straight edges, approximated by surface (a); one half of its $y z$ cross-section at an arbitrary point $x$ (b).

The parametric equation of line $a$, connecting points $\left(0,0, \frac{h}{2}\right)$ and $\left(L,-\frac{h}{2}, 0\right)$, is obvious (see Figure 2a):

$$
x=\xi L, \quad y=-\xi \frac{h}{2}, \quad z=(1-\xi) \frac{h}{2} .
$$

The angle of rotation, $\psi(x)$, rotates the vector $\vec{g}$, parallel to the $z$-axis

$$
\vec{g}=\left(0, \frac{h}{2}\right)
$$

into vector $\vec{G}$ :

$$
\vec{G}=\left(-\xi \frac{h}{2},(1-\xi) \frac{h}{2}\right)
$$


$\vec{G}$ is a radius vector of an edge point at an arbitrary value $x=\xi L$. Parameter $\xi$ is expressed by $\xi=\frac{x}{L}$ and inserted into (2):

$$
\vec{G}=\left(-\frac{h}{2 L} x,\left(1-\frac{x}{L}\right) \frac{h}{2}\right) .
$$

By scalarly multiplying $\vec{g}$ and $\vec{G}$ we then have

$$
\begin{aligned}
\vec{g} \cdot \vec{G} & =\|\vec{g}\|\|\vec{G}\| \cos \psi(x) \\
\cos \psi(x) & =\frac{L-x}{\sqrt{2 x^{2}-2 L x+L^{2}}} .
\end{aligned}
$$

Thus, the non-linear twist angle of a pre-twisted beam with straight edges reads

$$
\psi(x)=\arccos \frac{L-x}{\sqrt{2 x^{2}-2 L x+L^{2}}} .
$$

The difference with respect to the linear pre-twist case is now obvious; graphs of both functions are compared in Figure 3a.

The non-linear twist also affects the initial torsional pseudo-curvature of the beam. While the pseudo-curvature of the linear pre-twist is constant, $\frac{\pi}{2 L}$, the pseudo-curvature in the non-linear case is a rational function of $x$ (see Figure $3 \mathrm{~b})$

$$
\kappa(x)=\psi^{\prime}(x)=\frac{L}{2 x^{2}-2 L x+L^{2}} .
$$

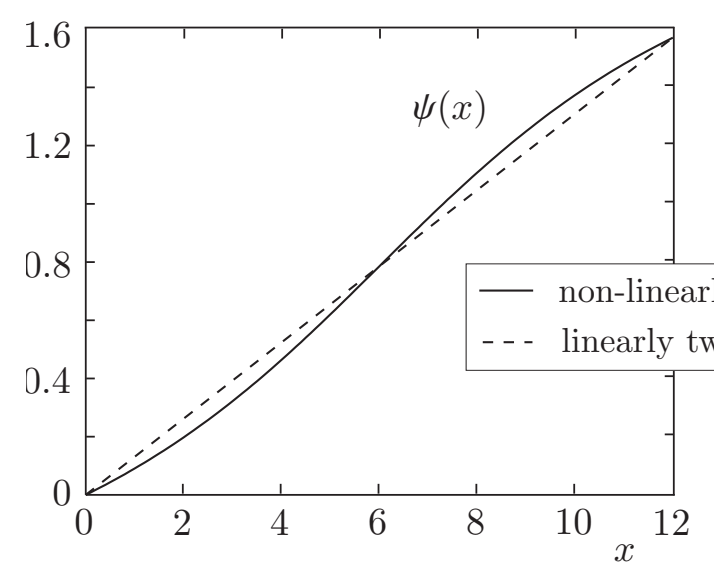

a)

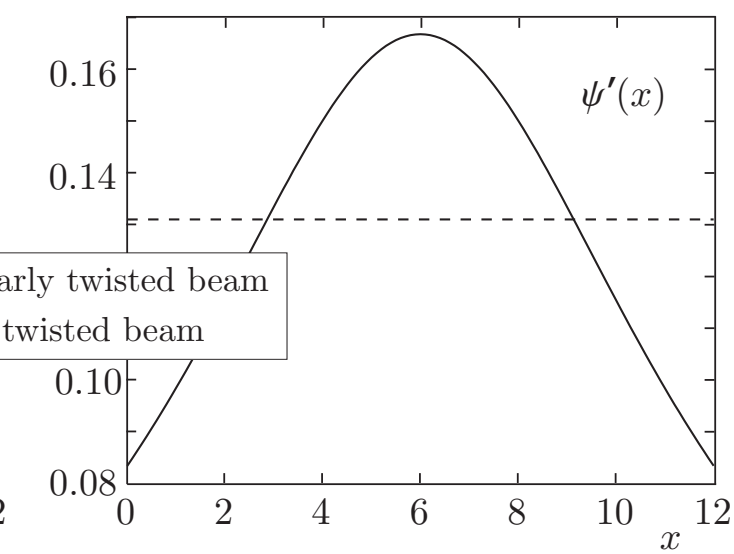

b)

Figure 3. Angle of twist and its derivative (torsional pseudo-curvature) along the axis of the beam.

We have thus deduced that the beam with straight edges must have the nonlinear twist angle $\psi(x)$ defined by (3). But it is then obvious that the width of 
the beam, $w$, must vary with $x$. By observing Figure $2 \mathrm{~b}$, we see that one half of the width of the cross-section at an arbitrary value of $x$ equals the length of vector $\vec{G}$ :

$$
w(x)=2\|\vec{G}\|=h \sqrt{2\left(\frac{x}{L}\right)^{2}-2 \frac{x}{L}+1} .
$$

Thus, if we use the beam with straight edges for the modeling of the pretwisted beam, we must take the width of the cross-sections along the beam to vary as described by (4). The graph of the width function, $w(x)$, of the beam with straight edges is shown in Figure 4. A considerable reduction of the beam width may be noticed, particularly at the mid-point of the beam, where the width is $\frac{h}{\sqrt{2}} \approx 0.78 h$.

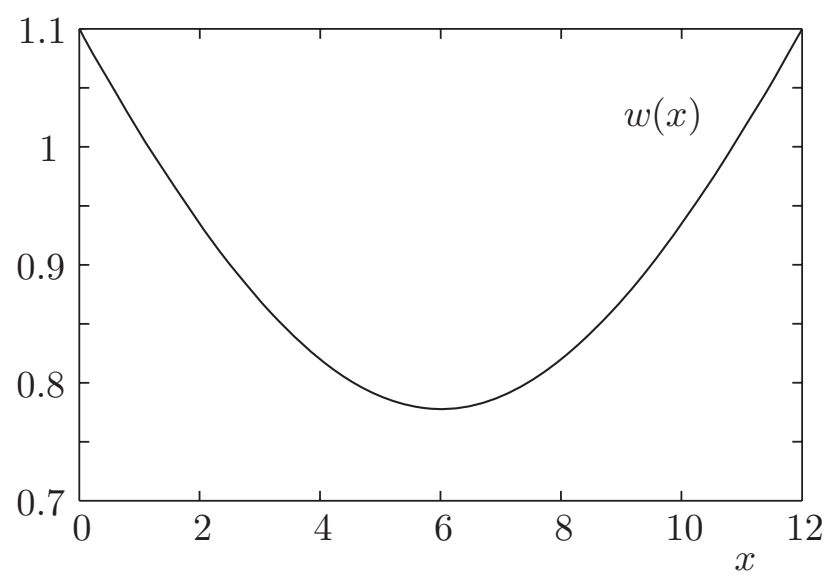

Figure 4 . The variable width of the beam with straight edges.

Please note that by taking the non-linear twist angle (3) (as in the straightedge case), but assuming constant width of the cross-sections, we again obtain the beam with curved edges whose shape is very similar, but not equal to the one given in Figure 1a.

Three types of initial beam shapes are thus plausible: (i) with the linear twist; (ii) with the non-linear twist and the constant width; and (iii) with the nonlinear twist and the variable width.

\section{Numerical results and comparison}

Since the three possible types of initial beam geometries have now been precisely defined, we can assess the effects of different initial shapes by the numerical solution of the problem. For the easier further comparison and discussion, let us present the numerical results of Dutta and White [2], the results of the shell theories [3-9], and the results of the three-dimensional theory of Lo 
Table 1

Free-end displacements of the twisted beam.

\begin{tabular}{|c|c|c|c|c|c|}
\hline \multirow[b]{2}{*}{ author } & \multirow[b]{2}{*}{$n_{e}$} & \multicolumn{2}{|c|}{ load case 1} & \multicolumn{2}{|c|}{ load case 2} \\
\hline & & displacement $u_{z}$ & error $(\%)$ & displacement $u_{y}$ & error $(\%)$ \\
\hline exact [1] & & 0.005424 & & 0.001754 & \\
\hline$[2]$ & 12 & 0.005402 & 0.41 & 0.001741 & 0.74 \\
\hline \multirow[t]{2}{*}[3]{} & $12 \times 2$ & 0.005348 & 1.40 & & \\
\hline & $14 \times 2$ & 0.005462 & 0.70 & & \\
\hline$[4]$ & $12 \times 2$ & 0.005587 & 3.00 & 0.001754 & 0.00 \\
\hline \multirow[t]{3}{*}[5]{} & $12 \times 2$ & & & 0.001717 & 2.10 \\
\hline & $24 \times 2$ & & & 0.001742 & 0.70 \\
\hline & $48 \times 4$ & & & 0.001747 & 0.40 \\
\hline \multirow[t]{3}{*}[6]{} & $12 \times 2$ & 0.005405 & 0.35 & 0.001755 & 0.06 \\
\hline & $24 \times 4$ & 0.005412 & 0.22 & 0.001753 & 0.06 \\
\hline & $48 \times 8$ & 0.005416 & 0.15 & 0.001752 & 0.11 \\
\hline \multirow[t]{2}{*}[7]{} & $12 \times 2$ & 0.005405 & 0.35 & 0.001754 & 0.00 \\
\hline & $24 \times 4$ & 0.005411 & 0.24 & 0.001751 & 0.17 \\
\hline$[8]$ & $12 \times 2$ & 0.005402 & 0.41 & 0.001753 & 0.04 \\
\hline \multirow[t]{2}{*}[9]{} & $12 \times 2$ & 0.005397 & 0.50 & 0.001727 & 1.54 \\
\hline & $48 \times 8$ & 0.005416 & 0.15 & 0.001751 & 0.17 \\
\hline [10] & $6 \times 2 \times 1$ & 0.005387 & 0.68 & 0.001724 & 1.71 \\
\hline
\end{tabular}

and Ling [10]. For each particular load case, the free-end displacements in the direction of the applied force are shown in Table 1, together with their absolute relative error with respect to [1].

The results in [2-10] compare well, which indicates that probably the same initial shape was used as in [1]. Yet this is not in accord with the geometric shapes of the twisted beams displayed in the papers by these authors. While in $[2-10]$ the shapes fully agree with the one in Figure $1 \mathrm{~b}$, the shape of the beam, presented in [1], looks more like the one in Figure 1a. Most of the results are relatively accurate, the relative error being lower than $1 \%$. Note, however, that only recent and therefore very accurate finite-element formulations are cited in Table 1. Because of the extreme accuracy of these sophisticated finite- 
Table 2

Free-end displacements of a twisted beam. Results of the beam element proposed by Zupan and Saje ([13], [14]).

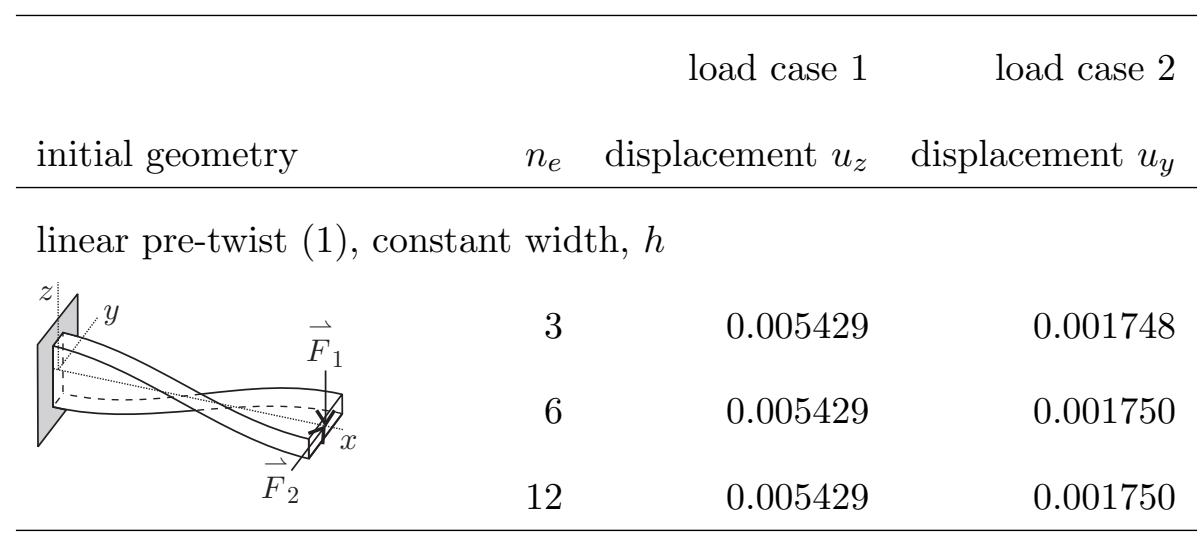

non-linear pre-twist (3), constant width, $h$

\begin{tabular}{|cccc}
$\vec{F}_{\vec{F}_{2}}$ & 3 & 0.005537 & 0.001640 \\
\hdashline & 6 & 0.005538 & 0.001641 \\
\hdashline & 12 & 0.005538 & 0.001641
\end{tabular}

non-linear pre-twist (3), variable width (4), w(x)

\begin{tabular}{|c|c|c|c|}
\hline$\vec{F}_{1}$ & 3 & 0.006716 & 0.002386 \\
\hline$\vec{x} y_{x}$ & 6 & 0.006717 & 0.002388 \\
\hline$F_{2}$ & 12 & 0.006717 & 0.002388 \\
\hline exact [1] & & 0.005424 & 0.001754 \\
\hline
\end{tabular}

$n_{e}=$ number of elements

elements, we would assume that the differences in results could stem only from the different initial geometric shapes taken in [2-10] and [1].

In order to resolve the issue, we compared their results to ours, obtained by our curvature-based finite-element formulation ([13]-[14]). This formulation uses the 'geometrically exact finite-strain beam theory' of Reissner [11] and Simo [12] and employs the incremental pseudo-curvature vector as the only interpolated variable. All three variants of initially twisted beams, described in previous section, were analyzed: (i) the linearly twisted beam, (ii) the non- 
linearly twisted beam with constant width, and (iii) the non-linearly twisted beam with variable width. Each case was modeled by three different finite element meshes with 3, 6 and 12 equally long linear elements. Our results are shown in Table 2.

The results show that the difference in the initial geometric shape affects the beam deformation. From Table 2 we observe that our results for the linearly twisted beam almost perfectly agree with [1]. The relative error is $0.09 \%$ for the first and $0.23 \%$ for the second load case. On the other hand, our results for the beam with straight edges are different in all non-zero digits. The relative differences are as big as $23.8 \%$ for the first and $36.1 \%$ for the second load case. The results for the non-linearly pre-twisted beam and constant width are different, too, yet the differences are very small. This again suggests that the results in [1] are meant for the linearly twisted (curved-edge) beam.

\section{Conclusion}

Test problems are of utmost importance in assessing the accuracy of newly proposed finite elements. One of such test problems is "the twisted beam problem" introduced by MacNeal and Harder [1]. The results of many finite-element developers indicate that the initial shape of the twisted beam as presented in [1] can be understood in different ways. Our analysis suggests that the twisted beam problem [1] assumes the linearly varying twist angle, which results in non-linearly shaped edges of the undeformed beam.

\section{References}

[1] R. H. MacNeal, R. L. Harder, "A proposed standard set of problems to test finite element accuracy", Finite Elem. Anal. Design 1, 3-20, 1985.

[2] A. Dutta, D. W. White, "Large displacement formulation of three-dimensional beam element with cross-sectional warping”, Comput. Struct. 45, 9-24, 1992.

[3] J. H. Argyris, M. Papadrakakis, C. Apostolopoulou, S. Koutsourelakis, "The TRIC shell element: theoretical and numerical investigation", Comput. Methods Appl. Mech. Eng. 182, 217-245, 2000.

[4] K. Bassayya, U. Shrinivasa, "A 14-node brick element, PN5X1, for plates and shells", Comput. Struct. 74, 167-178, 2000.

[5] P. Betsch, F. Gruttmann, E. Stein, "A 4-node finite shell element for the implementation of general hyperelastic 3D-elasticity at finite strains", Comput. Methods Appl. Mech. Eng. 130, 57-79, 1996. 
[6] A. A. Groenwold, N. Stander, "An efficient 4-node 24 dof thick shell finite element with 5-point quadrature", Eng. Computations 12, 723-747, 1995.

[7] A. Ibrahimbegovic, F. Frey, "Stress resultant geometrically non-linear shell theory with drilling rotations. Part III: linearized kinematics", Int. J. Numer. Methods Eng. 37, 3659-3683, 1994.

[8] J. Liu, H. R. Riggs, A. Tessler, "A four-node, shear-deformable shell element developed via explicit Kirchhoff constraints", Int. J. Numer. Methods Eng. 49, 1065-1086, 2000.

[9] Y. Zhu, T. Zacharia, "A new one-point quadrature, quadrilateral shell element with drilling degrees of freedom", Comput. Methods Appl. Mech. Eng. 136, 165203, 1996.

[10] S. H. Lo, C. Ling, "Improvement on the 10-node tetrahedral element for threedimensional problems", Comput. Methods Appl. Mech. Eng. 189, 961-974, 2000.

[11] E. Reissner, "On finite deformation of space-curved beams", J. Appl. Math. Phys. 32, 734-744, 1981.

[12] J. C. Simo, "A finite strain beam formulation. The three-dimensional dynamic problem. Part I", Comput. Methods Appl. Mech. Eng. 49, 55-70, 1985.

[13] D. Zupan, M. Saje, "A new finite element formulation of three-dimensional beam theory based on interpolation of curvature", CMES: Computer Modeling in Engineering and Sciences 4, No. 2, 301-318, 2003.

[14] D. Zupan, M. Saje, "The three-dimensional beam theory: finite element formulation based on curvature", Comput. Struct. 81, 1875-1888, 2003. 